\title{
HEAVY HADRON SPECTROSCOPY
}

\author{
ANTONIO VAIRO \\ Dipartimento di Fisica dell'Università di Milano and INFN, \\ via Celoria 16, 20133 Milano, Italy \\ E-mail: antonio.vairo@mi.infn.it
}

I review recent theoretical advances in heavy hadron spectroscopy.

Keywords: heavy quarks, quarkonium, new charmonium spectroscopy

\section{Introduction}

After having played a major role in the foundation of QCD, heavy hadron spectroscopy has witnessed in the last years a renewal of interest led by the many new data coming from the B factories, CLEO and the Tevatron and by the progress made in the theoretical methods. I will summarize the former and mostly focus on the latter. Much of the theoretical progress in the physics of heavy hadrons comes from effective field theories (EFTs) and lattice gauge theories. For most of these systems, they allow systematic treatments, which may be used to gain control over one of the most elusive sectors of the Standard Model, low-energy QCD, even in one of its most spectacular manifestations: the formation of exotic bound states.

A systematic treatment of heavy-quark bound states is possible because the systems are characterized by at least two small parameters. One is the strong coupling constant at the heavy-quark mass scale, $m$, which is, by definition, larger than the typical hadronic scale, $\Lambda_{\mathrm{QCD}}$, and the other is the ratio $\Lambda_{\mathrm{QCD}} / m$. Expansions in these two parameters can be exploited in the description of systems made by one heavy quark, like heavy-light mesons or baryons. If the expansions are made manifest at the Lagrangian level, the resulting EFT is known as Heavy Quark Effective Theory, HQET [1] (for some reviews see [2]).
Systems made by two heavy quarks are most frequently and successfully studied as non-relativistic bound states [3]. They are characterized by another small parameter, the heavy-quark velocity $v$, which comes with a hierarchy of energy scales: $m v, m v^{2}$, ... Making explicit at the Lagrangian level the expansions in $m v / m$ and $m v^{2} / m$ leads to an EFT known as non-relativistic QCD (NRQCD) [4, 5]. This EFT is similar to HQET, but with a different power counting. It also accounts for contact interactions between quarks and antiquarks (e.g. in decay processes) and hence has a wider set of operators. Making explicit at the Lagrangian level the expansion in $m v^{2} / m v$ leads to another EFT known as potential NRQCD (pNRQCD) [6] (an alternative EFT is in [7]). pNRQCD is close to a Schrödinger-like description of the bound state and hence as simple. The bulk of the interaction is carried by potential-like terms, but non-potential interactions, associated with the propagation of low-energy degrees of freedom, are generally present as well. For a review on nonrelativistic EFTs we refer to [8].

It is important to establish when $\Lambda_{\mathrm{QCD}}$ sets in, i.e. when we have to resort to nonperturbative methods. In the case of systems made by one heavy quark, $\Lambda_{\mathrm{QCD}}$ becomes the most relevant scale once the heavy-quark mass has been integrated out: all HQET matrix elements are non-perturbative. The situ- 
ation is more variegated in systems made by two heavy quarks. For low-lying resonances, it is reasonable, although not proved, to assume $m v^{2} \gtrsim \Lambda_{\mathrm{QCD}}$. The system is weakly coupled and we may rely on perturbation theory, for instance, to calculate the potential. The theoretical challenge here is performing higher-order calculations and the goal is precision physics. For high-lying resonances, we assume $m v \sim \Lambda_{\mathrm{QCD}}$. The system is strongly coupled and the potential must be determined non-perturbatively, for instance, on the lattice. The theoretical challenge here is providing a consistent framework where to perform lattice calculations and the progress is measured by the advance in lattice computations.

For what concerns systems close or above the open flavor threshold, a complete and satisfactory understanding of the dynamics has not been achieved so far. Hence, the study of these systems is on a less secure ground than the study of states below threshold. Although in some cases one may develop an EFT owing to special dynamical conditions (an example that we will discuss in the following is the $X(3872)$ interpreted as a loosely bound $D^{0} \bar{D}^{* 0}+$ $\bar{D}^{0} D^{* 0}$ molecule), the study of these systems largely relies on phenomenological models. The major theoretical challenge here is to interpret the new states in the charmonium region discovered at the B-factories in the last years (for an updated list, see the Quarkonium Working Group (QWG) page http://www.qwg.to.infn.it/).

In the following, I will discuss from a theoretical perspective advances in heavy hadron spectroscopy in the light of the results presented at ICHEP 2006. For charm and charmonium in experiments I refer to $[9$, 10], for light quark spectroscopy to [11]. An overview on the newly discovered charmonium resonances from an experimental perspective can be found in [12].

\section{Heavy-quark masses}

The heavy-quark masses are among the fundamental parameters of the Standard Model that may be extracted with precision from heavy-light or heavy-heavy quark systems. Different recent determinations of the $c$ mass (from the $J / \psi, \bar{m}_{c}\left(\bar{m}_{c}\right)=1.24 \pm 0.02 \mathrm{GeV}$ [13], from the $D, \bar{m}_{c}\left(\bar{m}_{c}\right)=1.21 \pm 0.1$ $\mathrm{GeV}$ [14], from high moments sum rules, $\bar{m}_{c}\left(\bar{m}_{c}\right)=1.19 \pm 0.11 \mathrm{GeV}[15]$, from inclusive $B$ decays, $\bar{m}_{c}\left(\bar{m}_{c}\right)=1.224 \pm 0.06 \mathrm{GeV}$ [16], from low moments sum rules, $\bar{m}_{c}\left(\bar{m}_{c}\right)=$ $1.290 \pm 0.015 \mathrm{GeV}[17]$ ) and the $b$ mass (from the $\Upsilon(1 S), \bar{m}_{b}\left(\bar{m}_{b}\right)=4.19 \pm 0.03 \mathrm{GeV}[18]$, $\bar{m}_{b}\left(\bar{m}_{b}\right)=4.346 \pm 0.070 \mathrm{GeV}[19], \bar{m}_{b}\left(\bar{m}_{b}\right)=$ $4.20 \pm 0.04 \mathrm{GeV}[20], \bar{m}_{b}\left(\bar{m}_{b}\right)=4.24 \pm 0.07$ $\mathrm{GeV}$ [21], from $B \rightarrow X_{s} \gamma, \bar{m}_{b}\left(\bar{m}_{b}\right)=4.22 \pm$ $0.09 \mathrm{GeV}[22]$, from high moments sum rules, $\bar{m}_{b}\left(\bar{m}_{b}\right)=4.19 \pm 0.06 \mathrm{GeV}[23]$, from low moments sum rules, $\bar{m}_{b}\left(\bar{m}_{b}\right)=4.18 \pm 0.035 \mathrm{GeV}$ [17]) show a remarkable agreement (with the possible exception of [19], for a critical discussion we refer to [3]).

\section{Heavy-light mesons and baryons}

$\bar{Q} q$ and $Q q q$ systems are characterized by the hierarchy of scales $m \gg \Lambda_{\mathrm{QCD}}$, where the typical distance of the heavy quark from the light ones is of order $1 / \Lambda_{\mathrm{QCD}}$. Theoretical advances in the calculation of heavy hadron lifetimes in the framework of HQET have been presented in [24].

In the last year, new $D_{s J}$ searches have led to the discovery of new resonances at BELLE, $D_{s J}(2700), \Gamma \approx 115 \mathrm{MeV}$ [25], and at BABAR, $D_{s J}(2856), \Gamma \approx 47 \mathrm{MeV}$ [26], and new $B_{s J}$ searches to the discovery of a new resonance at CDF, $B_{s 1}(5829)$ [27]. $c q q$ baryons have been investigated at BABAR leading to the discovery of the $\Lambda_{c}(2940), \Gamma \approx$ $17 \mathrm{MeV}[28,29]$ and at BELLE leading to the discovery of the $\Xi_{c}(2980)^{+}, \Gamma \approx 43.5 \mathrm{MeV}$ and the $\Sigma_{c}(3077)^{+}, \Gamma \approx 5.2 \mathrm{MeV}$ [30]. 


\section{Low-lying $Q \bar{Q}$}

Low-lying $Q \bar{Q}$ states are expected to realize the hierarchy: $m \gg m v \gg m v^{2} \gtrsim \Lambda_{\mathrm{QCD}}$, where $m v$ is the typical scale of the inverse distance between the heavy quark and antiquark and $m v^{2}$ the typical scale of the binding energy. At a scale $\mu$ such that $m v \gg \mu \gg$ $m v^{2}$ the effective degrees of freedom are $Q \bar{Q}$ states (in color singlet and octet configurations), low-energy gluons and light quarks.

The lowest-lying quarkonium states are $\eta_{b}$ (not yet detected), $\Upsilon(1 S), \eta_{c}, J / \psi, B_{c}$ and $B_{c}^{*}$ (not yet detected). As mentioned above, the $\Upsilon(1 S)$ and $J / \psi$ masses may be used to extract the bottom and charm quark masses. Once the heavy-quark masses are known, one may use them for the determination of quarkonium ground-state observables. At NNLO the $B_{c}$ mass was calculated in [31] $\left(M_{B_{c}}=6326 \pm 29 \mathrm{MeV}\right)$, [13] $\left(M_{B_{c}}=6324 \pm 22 \mathrm{MeV}\right)$ and [18] $\left(M_{B_{c}}=\right.$ $6307 \pm 17 \mathrm{MeV})$. These values agree well with the unquenched lattice determination of [32] $\left(M_{B_{c}}=6304 \pm 12_{-0}^{+18} \mathrm{MeV}\right)$, which shows that the $B_{c}$ mass is not very sensitive to non-perturbative effects. This is confirmed by a recent measurement of the $B_{c}$ in the channel $B_{c} \rightarrow J / \psi \pi$ by the CDF collaboration at the Tevatron; they obtain with $360 \mathrm{pb}^{-1}$ of data $M_{B_{c}}=6285.7 \pm 5.3 \pm$ $1.2 \mathrm{MeV}$ [33], while the latest available figure based on $1.1 \mathrm{fb}^{-1}$ of data is $M_{B_{c}}=$ $6276.5 \pm 4.0 \pm 2.7 \mathrm{MeV}$ (see http://wwwcdf.fnal.gov/physics/new/bottom/060525.blessed-bc-mass/).

The bottomonium (and charmonium) ground-state hyperfine splitting has been calculated at NLL in [34]. Combining it with the measured $\Upsilon(1 S)$ mass, this determination provides a quite precise prediction for the $\eta_{b}$ mass: $M_{\eta_{b}}=9421 \pm 10_{-8}^{+9} \mathrm{MeV}$, where the first error is an estimate of the theoretical uncertainty and the second one reflects the uncertainty in $\alpha_{\mathrm{s}}$. Note that the discovery of the $\eta_{b}$ may provide a very competitive source of $\alpha_{\mathrm{s}}$ at the bottom mass scale with a projected error at the $M_{Z}$ scale of about 0.003. Similarly, in [35], the hyperfine splitting of the $B_{c}$ was calculated at NLL accuracy: $M_{B_{c}^{*}}-M_{B_{c}}=65 \pm 24_{-16}^{+19} \mathrm{MeV}$.

The ratios of electromagnetic decay widths were calculated for the ground state of charmonium and bottomonium at NNLL order in [36]; for the latter the result is $\Gamma\left(\eta_{b} \rightarrow \gamma \gamma\right) / \Gamma\left(\Upsilon(1 S) \rightarrow e^{+} e^{-}\right)=0.502 \pm$ $0.068 \pm 0.014$. A partial NNLL order analysis for the absolute width of $\Upsilon(1 S) \rightarrow e^{+} e^{-}$ can be found in [37].

Allowed magnetic dipole transitions between charmonium and bottomonium ground states have been considered at NNLO in $[38,39]$. The results are: $\Gamma(J / \psi \rightarrow$ $\left.\gamma \eta_{c}\right)=(1.5 \pm 1.0) \mathrm{keV}$ and $\Gamma\left(\Upsilon(1 S) \rightarrow \gamma \eta_{b}\right)$ $=\left(k_{\gamma} / 39 \mathrm{MeV}\right)^{3}(2.50 \pm 0.25) \mathrm{eV}$, where the errors account for uncertainties coming from higher-order corrections. The width $\Gamma\left(J / \psi \rightarrow \gamma \eta_{c}\right)$ is consistent with the PDG value [40]. Concerning $\Gamma\left(\Upsilon(1 S) \rightarrow \gamma \eta_{b}\right)$, a photon energy $k_{\gamma}=39 \mathrm{MeV}$ corresponds to a $\eta_{b}$ mass of $9421 \mathrm{MeV}$.

The radiative transition $\Upsilon(1 S) \rightarrow \gamma X$ has been considered in $[41,42]$. The agreement with the CLEO data [43] is very good.

\section{Low-lying $Q Q q$}

The SELEX collaboration at Fermilab reported evidence of five resonances that may be possibly identified with doubly charmed baryons [44]. Although these findings have not been confirmed by other experiments (notably by BELLE [45] and BABAR [46, 29]) they have triggered a renewed theoretical interest in doubly heavy baryon systems.

Low-lying $Q Q q$ states are expected to realize the hierarchy: $m \gg m v \gg \Lambda_{\mathrm{QCD}}$, where $m v$ is the typical inverse distance between the two heavy quarks and $\Lambda_{\mathrm{QCD}}$ is the typical inverse distance between the centerof-mass of the two heavy quarks and the light quark. 
At a scale $\mu$ such that $m v \gg \mu \gg \Lambda_{\mathrm{QCD}}$ the effective degrees of freedom are $Q Q$ states (in color antitriplet and sextet configurations), low-energy gluons and light quarks. Since the system shares features of heavylight mesons and quarkonia, the most suitable EFT at the scale $\mu$ is a combination of pNRQCD and HQET [47, 48]. The hyperfine splittings of the doubly heavy baryon lowest states have been calculated at NLO in $\alpha_{\mathrm{s}}$ and at $\mathrm{LO}$ in $\Lambda_{\mathrm{QCD}} / m$ by relating them to the hyperfine splittings of the $D$ and $B$ mesons (this method was first used in [49]). In [47], the obtained values are: $M_{\Xi_{c c}^{*}}-M_{\Xi_{c c}}=120 \pm 40$ $\mathrm{MeV}$ and $M_{\Xi_{b b}^{*}}-M_{\Xi_{b b}}=34 \pm 4 \mathrm{MeV}$, which are consistent with the quenched lattice determinations of $[50,51,52,53]$. Chiral corrections to the doubly heavy baryon masses, strong decay widths and electromagnetic decay widths have been considered in [54].

Also low-lying $Q Q Q$ baryons can be studied in a weak coupling framework. Three quark states can combine in four color configurations: a singlet, two octets and a decuplet, which lead to a rather rich dynamics [47]. Masses of various $Q Q Q$ ground states have been calculated with a variational method in [55]: since baryons made of three heavy quarks have not been discovered so far, it may be important for future searches to remark that the baryon masses turn our to be lower than those generally obtained in strong coupling analyses.

\section{High-lying $Q \bar{Q}$}

High-lying $Q \bar{Q}$ states are expected to realize the hierarchy: $m \gg m v \sim \Lambda_{\mathrm{QCD}} \gg m v^{2}$. Since we cannot measure directly $m v$, we do not know exactly where the border between low-lying and high-lying states lies. This difficulty is reflected in the literature. A weakcoupling treatment for the lowest-lying bottomonium masses ( $n=1, n=2$ states and the $\Upsilon(3 S)$ ) works well at NNLO in [13] and at $\mathrm{N}^{3} \mathrm{LO}$ in [56]. The outcome is more am- biguous for the fine splittings of the bottomonium $1 P$ levels in the NLO analysis of [57] and is positive only for the $\Upsilon(1 S)$ mass in the $\mathrm{N}^{3} \mathrm{LO}$ analysis of [58]. In the weak-coupling regime, the magnetic-dipole hindered transition $\Upsilon(2 S) \rightarrow \gamma \eta_{b}$ at leading order [38] does not agree with the experimental upper bound [59], while the ratios for different $n$ of the radiative decay widths $\Gamma(\Upsilon(n S) \rightarrow \gamma X)$ are better consistent with the data if the $\Upsilon(1 S)$ is assumed to be a weakly-coupled bound state and the $\Upsilon(2 S)$ and $\Upsilon(3 S)$ strongly coupled ones [60].

Masses of high-lying quarkonia may be accessed by lattice calculations. A recent unquenched QCD determination of the charmonium spectrum below the open flavor threshold with staggered sea quarks may be found in [61]. At present, bottomonium is too heavy to be implemented directly on the lattice. A solution is provided by NRQCD [62]. Since the heavy-quark mass scale has been integrated out, for NRQCD on the lattice, it is sufficient to have a lattice spacing $a$ as coarse as $m \gg 1 / a \gg m v$. A price to pay is that, by construction, the continuum limit cannot be reached. Another one is that the NRQCD Lagrangian has to be supplemented by matching coefficients calculated in lattice perturbation theory, which encode the contributions from the heavy-mass energy modes that have been integrated out. A recent unquenched determination of the bottomonium spectrum with staggered sea quarks can be found in [63]. The fact that all matching coefficients of NRQCD on the lattice are taken at their tree-level value induces a systematic error of order $\alpha_{\mathrm{s}} v^{2}$ for the radial splittings and of order $\alpha_{\mathrm{s}}$ for the fine and hyperfine splittings.

At a scale $\mu$ such that $m v \sim \Lambda_{\mathrm{QCD}} \gg$ $\mu \gg m v^{2}$, confinement sets in. Below threshold, the effective degrees of freedom are $Q \bar{Q}$ states (in color singlet configuration) and light quarks. Without light quarks, the $Q \bar{Q}$ interaction would be simply described by a 
non-relativistic potential [64, 65]. The potential is in general a complex valued function admixture of perturbative terms, inherited from NRQCD, which encode highenergy contributions, and non-perturbative ones. The latter may be expressed in terms of Wilson loops and, therefore, are well suited for lattice calculations.

The real part of the potential has been one of the first quantities to be calculated on the lattice (for a review see [66]). In the last year, there has been some remarkable progress [67]. In [68], the $1 / m$ potential has been calculated for the first time. The existence of this potential was first pointed out in the pNRQCD framework [64]. The lattice result shows that the potential has a $1 / r$ behaviour, which, in the charmonium case, is of the same size as the $1 / r$ Coulomb tail of the static potential and, in the bottomonium one, is about $25 \%$. Therefore, if the $1 / m$ potential is to be considered part of the leading-order quarkonium potential, then the latter would turn out to be, somewhat surprisingly, a flavor-dependent function. In [69], spin-dependent potentials have been calculated with unprecedented precision. In the long range, the spin-orbit potentials show, for the first time, deviations from the flux-tube picture of chromoelectric confinement [70], which is predicted in many models of the QCD vacuum [71]. Spin-spin and tensor potentials do not show sizeable long-range contributions. In the data, this is reflected, for instance, by the smallness of the $P$-wave hyperfine splitting: the E835 experiment measures a $h_{c}$ mass $M_{h_{c}}=3525.8 \pm$ $0.2 \pm 0.2 \mathrm{MeV}(\Gamma<1 \mathrm{MeV})[72]$ and CLEO measures $M_{h_{c}}=3524.4 \pm 0.6 \pm 0.4 \mathrm{MeV}$ [73], both very close to the center-of-gravity mass $M_{\text {c.o.g. }}(1 P)=3525.36 \pm 0.2 \pm 0.2 \mathrm{MeV}$.

The expectation value of the imaginary part of the potential provides the quarkonium width. At the level of NRQCD, stateof-the art expressions for the decay widths may be found in $[74,75,76]$, at the level of
pNRQCD in $[77,78,79,80]$. Charmonium $P$-wave decay widths calculated in NRQCD [3] and bottomonium $P$-wave decay widths calculated in pNRQCD [77, 81] are consistent with the most recent data [3]. In both cases, analyses have been performed at NLO in $\alpha_{\mathrm{s}}$ and at leading order in the velocity expansion.

\section{Threshold states}

Most of the newly discovered resonances in the charmonium region are near or above threshold. A comprehensive review updated at January 2006 is [82]. For states near or above threshold a general and systematic treatment does not exist so far. Most of the existing analyses rely on models (e.g., for the coupling with two-mesons states, the Cornell coupled-channel model $[83,84]$ and the ${ }^{3} P_{0}$ model $[85,86])$. This makes it difficult to predict with precision even the masses of the states, which is usually not the case for states below threshold.

\subsection{Candidates for $c \bar{c}$ states}

Among the recently discovered signals that may be ascribed to traditional $c \bar{c}$ bound states are the $X(3940), Z(3930)$ and $Y(3940)$.

The state labeled $X(3940)$ has been seen in $e^{+} e^{-} \rightarrow J / \psi X$ by the BELLE collaboration [87] with a mass $M_{X}=3943 \pm 6 \pm 6 \mathrm{MeV}$ and a width $\Gamma<52 \mathrm{MeV}$. In [88], it has been suggested a $\eta_{c}(3 S)$ interpretation for the state, but then the mass would be somewhat lower to what expected in potential models. The state contributes to the large cross section of $e^{+} e^{-} \rightarrow J / \psi+c \bar{c}$, which turns out to be $(82 \pm 15 \pm 14) \%$ of the total cross section $e^{+} e^{-} \rightarrow J / \psi+X[89]$. Theoretical problems related to double charmonium production have been discussed in [90]. For recent progress see [91].

A state labeled $Z(3930)$ has been seen by the BELLE collaboration in $\gamma \gamma \rightarrow D \bar{D}$ [92] 
with a mass $M_{Z}=3929 \pm 5 \pm 2 \mathrm{MeV}$ and a width $\Gamma=29 \pm 10 \pm 2 \mathrm{MeV}$. So far, the properties of the state fit well with a $\chi_{c 2}(2 P)$ interpretation.

More enigmatic is the interpretation of the state $Y(3940)$ seen by the BELLE collaboration in $B \rightarrow K Y \rightarrow K \omega J / \psi$ [93]. The reported mass is $M_{Y}=3943 \pm 11 \pm 13 \mathrm{MeV}$ and the width is $\Gamma=87 \pm 22 \pm 26 \mathrm{MeV}$. The mass and width seem to fit with a radially excited $P$-wave charmonium, however the discovery decay process also suggests more exotic interpretations: for instance, a tetraquark interpretation has been proposed in [94].

Finally, at the QWG meeting of this year [95] and at this conference [96], the BABAR collaboration reported a new signal seen in $e^{+} e^{-} \rightarrow \psi(2 S) \pi \pi$. If identified with a resonance, labeled $Y(4350)$, its mass would be $M_{Y}=4354 \pm 16 \mathrm{MeV}$ and its decay width $\Gamma=106 \pm 19 \mathrm{MeV}$. So far, no theoretical interpretation has been put forward.

\subsection{Candidates for exotic states: $X(3872)$ and $Y(4260)$}

Some of the newly discovered states allow for exotic interpretations. This is particularly the case for the $X(3872)$ and the $Y(4260)$.

The state $X(3872)$ has been discovered by BELLE in $B^{ \pm} \rightarrow K^{ \pm} X \rightarrow K^{ \pm} \pi^{+} \pi^{-} J / \psi$ with $M_{X}=3872.0 \pm 0.6 \pm 0.5 \mathrm{MeV}[97$, 98], and confirmed by BABAR $[99,96]$ that measures $M_{X}=3871.3 \pm 0.6 \pm 0.1 \mathrm{MeV}$ in $B^{-} \rightarrow K^{-} \pi^{+} \pi^{-} J / \psi$ and $M_{X}=3868.6 \pm$ $1.2 \pm 0.2 \mathrm{MeV}$ in $B^{0} \rightarrow K^{0} \pi^{+} \pi^{-} J / \psi$ [100]. The state has also been seen at the Tevatron in $p \bar{p} \rightarrow X \rightarrow \pi^{+} \pi^{-} J / \psi$ by $\mathrm{CDF}$ with a mass $M_{X}=3871.3 \pm 0.7 \pm 0.4 \mathrm{MeV}$ [101, 102] and by D0 with a mass $M_{X}=$ $3871.8 \pm 3.1 \pm 3.0 \mathrm{MeV}$ [103]. BELLE has an upper limit on the width: $\Gamma<2.3 \mathrm{MeV}$. The $X(3872)$ has been detected in four different decay modes: $X \rightarrow \pi^{+} \pi^{-} J / \psi$, which is the discovery mode, $X \rightarrow \pi^{+} \pi^{-} \pi^{0} J / \psi$ [104], $X \rightarrow \gamma J / \psi$ [105] and $X \rightarrow D^{0} \bar{D}^{0} \pi^{0}$
[106]. The last one is likely to be the dominant decay mode: in [107], it was found that $\mathcal{B}\left(X \rightarrow \pi^{+} \pi^{-} J / \psi\right)>4.2 \%$ at $90 \%$ C.L., which, combined with the ratio $\mathcal{B}(X \rightarrow$ $\left.D^{0} \bar{D}^{0} \pi^{0}\right) / \mathcal{B}\left(X \rightarrow \pi^{+} \pi^{-} J / \psi\right)=9.4_{-4.3}^{+3.6}$ measured in [106], gives $\mathcal{B}\left(X \rightarrow D^{0} \bar{D}^{0} \pi^{0}\right)>$ $40_{-20}^{+15 \%}$. One should notice that BELLE finds a threshold enhancement peak in the $D^{0} \bar{D}^{0} \pi^{0}$ invariant mass at $3875.4 \pm 0.7_{-2.0}^{+1.2}$ $\mathrm{MeV}$, which is $2.0 \sigma$ larger than the worldaverage mass of the $X(3872)$. The decay mode $X \rightarrow \gamma J / \psi$ implies that the $X(3872)$ has positive charge conjugation. Analyses of angular distributions performed by BELLE [108] and CDF [109] favor a spin parity assignment $1^{+}$. The ratio $\mathcal{B}(X \rightarrow$ $\left.\pi^{+} \pi^{-} \pi^{0} J / \psi\right) / \mathcal{B}\left(X \rightarrow \pi^{+} \pi^{-} J / \psi\right)=1.0 \pm$ $0.4 \pm 0.3$ measured by BELLE [105] suggests that the $X(3872)$ is a mixture of isospin $I=1$ and $I=0$ states. The substantial $I=1$ component requires that the $X(3872)$ contains $u \bar{u} / d \bar{d}$ pairs in addition to hidden charm, which thus qualifies it as a fourquark state [110]. Hence, most recently, the majority of theoretical studies has analyzed the $X(3872)$ as a four-quark state with $J^{P C}$ quantum numbers $1^{++}$.

Three equivalent quark-pair configurations are possible for a four-quark state of the type $c \bar{c} q \bar{q}$ ( $q$ stands for a generic light quark). They have been all exploited in the literature. However, the resulting models are not equivalent, because different dynamics are attributed to different configurations. In [111], it is assumed that $X \sim$ $(c \bar{c})_{S=1}^{8}(q \bar{q})_{S=1}^{8}$, i.e. that the dominant Fockspace component contains a $c \bar{c}$ pair and a $q \bar{q}$ pair in a color octet configuration with spin 1. This configuration is equivalent to $X \sim(c \bar{q})_{S=0}^{1}(q \bar{c})_{S=1}^{1}+(c \bar{q})_{S=1}^{1}(q \bar{c})_{S=0}^{1}$, which is the molecular picture that we will discuss later. Calculations are based on the phenomenological interaction Hamiltonian $H=$ $-\sum_{i j} C_{i j} T^{a} \otimes T^{a} \boldsymbol{\sigma} \otimes \boldsymbol{\sigma} . \quad$ It is expected that decays into charmonium with light pseudoscalar mesons are suppressed with respect 
to decays into charmonium with light vector mesons; moreover, two neutral states made of $c u \bar{c} \bar{u}$ and $c d \bar{c} \bar{d}$ and two charged ones made of $c u \bar{c} \bar{d}$ and $c d \bar{c} \bar{u}$ should exist.

In [94], it is assumed that $X \sim$ $(c q)_{S=1}^{\overline{3}}(\bar{c} \bar{q})_{S=0}^{3}+(c q)_{S=0}^{\overline{3}}(\bar{c} \bar{q})_{S=1}^{3}$. Here, the clustering of quark pairs in tightly bound color triplet diquarks is not induced by a scale separation, like in the doubly heavy baryon case discussed above, but is a dynamical assumption of the model. Predictions are based on the phenomenological interaction Hamiltonian $H=\sum_{i j} \kappa_{i j} \boldsymbol{\sigma} \otimes \boldsymbol{\sigma}$. In particular, the model predicts the existence of two neutral states made of $c u \bar{c} \bar{u}$ and $c d \bar{c} \bar{d}$ and of two charged ones. The mass difference between the two neutral states should be $\Delta M_{X}=2\left(m_{d}-m_{u}\right) / \cos (2 \theta) \approx(8 \pm 3)$ $\mathrm{MeV}$ if the mixing angle $\theta$ is fixed on $\Gamma(X \rightarrow$ $\left.\pi^{+} \pi^{-} \pi^{0} J / \psi\right) / \Gamma\left(X \rightarrow \pi^{+} \pi^{-} J / \psi\right)$. Since $B^{ \pm} \rightarrow K^{ \pm} X$ and $B^{0} \rightarrow K^{0} X$ produce $c u \bar{c} \bar{u}$ and $c d \bar{c} \bar{d}$ in different amount, the mass difference should be seen in the two processes. The BABAR result [100], $\Delta M_{X}=(2.7 \pm 1.3 \pm 0.2)$ $\mathrm{MeV}$, is not conclusive. Searches for charged partners of the $X(3872)$ have been negative so far, while the $2^{++}$partner may be consistent with the $Y(3940)$. In the same framework, a tetraquark interpretation of the $D_{s}$ particles has been also advanced.

In $[112,113]$, it is assumed that $X \sim$ $(c \bar{q})_{S=0}^{1}(q \bar{c})_{S=1}^{1}+(c \bar{q})_{S=1}^{1}(q \bar{c})_{S=0}^{1} \sim D^{0} \bar{D}^{* 0}+$ $D^{* 0} \bar{D}^{0}$, i.e. that the dominant Fock-space component of the $X(3872)$ is a $D^{0} \bar{D}^{* 0}$ and $D^{* 0} \bar{D}^{0}$ molecule; small short-range components of the type $(c \bar{c})_{S=1}^{1}(q \bar{q})_{S=1}^{1} \sim J / \psi \rho, \omega$ are included as well. Predictions depend on the adopted phenomenological Hamiltonian, which typically contains, in the short range $\left(\sim 1 / \Lambda_{\mathrm{QCD}}\right)$, potential-type interactions among the quarks and, in the long range $\left(\sim 1 / m_{\pi}\right)$, the one-pion exchange. The prediction $\Gamma\left(X \rightarrow \pi^{+} \pi^{-} J / \psi\right) \approx$ $\Gamma\left(X \rightarrow \pi^{+} \pi^{-} \pi^{0} J / \psi\right)$ made in [113] turned out to be consistent with the BELLE result [105]. However, another prediction, $\Gamma(X \rightarrow$ $\left.\pi^{+} \pi^{-} J / \psi\right) \approx 20 \Gamma\left(X \rightarrow D^{0} \bar{D}^{0} \pi^{0}\right)$, is two orders of magnitude far from the data [106]. Not necessarily this points to a failure of the molecular model, but possibly to a smaller $J / \psi \rho$ component in the $X(3872)$ Fock space.

In $[114,115,116]$, it is assumed not only that the $X(3872)$ is a $D^{0} \bar{D}^{* 0}$ and $\bar{D}^{0} D^{* 0}$ molecule, but also that it is loosely bound, i.e. that the following hierarchy of scales is realized: $\Lambda_{\mathrm{QCD}} \gg m_{\pi} \gg m_{\pi}^{2} / M_{D_{0}} \approx$ $10 \mathrm{MeV} \gg E_{\text {binding. Indeed, the binding }}$ energy, $E_{\text {binding, which may be estimated }}$ from $M_{X}-\left(M_{D^{* 0}}+M_{D^{0}}\right)$, is very close to zero, i.e. much smaller than the natural scale $m_{\pi}^{2} / M_{D_{0}}$. This is also the case when using the new CLEO determination of the $D_{0}$ mass, $M_{D_{0}}=1864.85 \pm 0.15 \pm 0.20$ $\mathrm{MeV}$, [117]. The main uncertainty comes from the $X(3872)$ mass. Systems with a short-range interaction and a large scattering length have universal properties that may be exploited: in particular, production and decay amplitudes factorize in a short-range and a long-range part, where the latter depends only on one single parameter, the scattering length. A universal property that fits well with the observed large branching fraction of the $X(3872)$ decaying into $D^{0} \bar{D}^{0} \pi^{0}$ is $\mathcal{B}\left(X \rightarrow D^{0} \bar{D}^{0} \pi^{0}\right) \approx \mathcal{B}\left(D^{* 0} \rightarrow D^{0} \pi^{0}\right) \approx$ $60 \%$. This supports the view that the difficulties met in [113] to account for this decay channel may be specific of that model and not of the molecular picture in general.

The state $Y(4260)$ has been discovered by $\mathrm{BABAR}$ in the radiative return process $e^{+} e^{-} \rightarrow \gamma \pi^{+} \pi^{-} J / \psi$ with mass $M_{Y}=4259 \pm$ $8_{-6}^{+2} \mathrm{MeV}$ and width $\Gamma=88 \pm 23_{-4}^{+6} \mathrm{MeV}$ $[118,96]$, and seen in the same process by BELLE with mass $M_{Y}=4295 \pm 10_{-5}^{+11} \mathrm{MeV}$ and width $\Gamma=133 \pm 26_{-6}^{+13} \mathrm{MeV}[119,98]$ and by CLEO with mass $M_{Y}=4284_{-16}^{+17} \pm$ $4 \mathrm{MeV}$ and width $\Gamma=73_{-25}^{+39} \pm 5 \mathrm{MeV}$ [120]. CLEO has also confirmed the existence of an enhancement in the $\pi^{+} \pi^{-} J / \psi$ cross section at $4260 \mathrm{MeV}$ in a measurement of di- 
rect $e^{+} e^{-}$annihilation at $\sqrt{s}=4040,4160$ and $4260 \mathrm{MeV}[121,122]$. The $Y(4260)$ $J^{P C}$ quantum numbers are $1^{--}$. The $\pi^{+} \pi^{-}$ spectrum does not show signs of $f_{0}(980)$ or $f_{0}(600)$ [121]. BABAR measures $\mathcal{B}(Y \rightarrow$ $D \bar{D}) / \mathcal{B}\left(Y \rightarrow \pi^{+} \pi^{-} J / \psi\right)<7.6(\approx 500$ for $\psi(3770)$, which suggests an exotic interpretation for the $Y(4260))[123,96]$; BELLE sees a strong drop and local minimum of the $D^{*+} D^{*-}$ invariant mass at $4260 \mathrm{MeV}$ in $e^{+} e^{-} \rightarrow \gamma D^{*+} D^{*-}[124,98]$.

Many interpretations have been suggested for the $Y(4260): Y \sim \psi(4 S)$ [125], $Y \sim \Lambda_{c} \bar{\Lambda}_{c}[126], Y \sim\left[(c s)_{S=0}^{\overline{3}}(\bar{c} \bar{s})_{S=0}^{3}\right]_{\mathrm{P}-\text { wave }}$

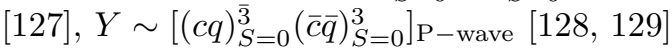
$Y \sim \chi_{c 1} \rho$ [130], $Y \sim \chi_{c 1} \omega$ [131] and $Y$ as a charmonium hybrid $[128,132,133]$. In particular, if interpreted as a charmonium hybrid, one may rely on the heavy-quark expansion and on lattice calculations to study its properties. Decays into $D^{(*)} \bar{D}^{(*)}$ should be suppressed, since they are forbidden at leading order in the heavy-quark expansion [132] (in the tetraquark picture [127] the most natural decay should be into $\left.D_{s} \bar{D}_{s}\right)$. This is in agreement with the upper limit on $Y \rightarrow D \bar{D}$ reported by BABAR and calls for an understanding of the signal seen by BELLE in $e^{+} e^{-} \rightarrow \gamma D^{*+} D^{*-}$. It is suggestive also that, according to lattice calculations [134], the lowest hybrid state is expected to contain a pseudoscalar color-octet quark-antiquark pair and gluons, whose quantum numbers are those of the electric cloud in a diatomic

$\Pi_{u}$ molecule, so that, indeed, the system has $J^{P C}$ numbers $1^{--}$, like the $Y$.

\section{3. $X$ and $Y$ in the bottomonium region}

States analogous to the $X(3872)$ and the $Y(4260)$ could also exist in the bottomonium region. Their finding could provide a confirmation of their interpretations in a more controlled framework for the theory. Possibilities for searches of these states have been discussed in [135].

In the framework of the molecular picture, in [136], a $1^{++} B^{0} \bar{B}^{* 0}$ and $B^{* 0} \bar{B}^{0}$ molecular state is predicted at 10604 $\mathrm{MeV}$. In [129], the $1^{++}(b q)_{S=1}^{\overline{3}}(\bar{b} \bar{q})_{S=0}^{3}+$ $(b q)_{S=0}^{\overline{3}}(\bar{b} \bar{q})_{S=1}^{3}$ tetraquark is predicted at $10492 \mathrm{MeV}$. The lattice determination [137] predicts the lowest bottomonium hybrid at a mass of $11020 \pm 180 \mathrm{MeV}$ (solving the Schrödinger equation for the $\Pi_{u}$ static energy calculated in [134] at intermediate distances gives a mass of about $10750 \mathrm{MeV})$. In [129],

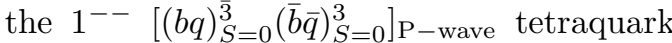
is predicted at $10807 \mathrm{MeV}$ and the $1^{--}$ $\left[(b s)_{S=0}^{\overline{3}}(\bar{b} \bar{s})_{S=0}^{3}\right]_{\mathrm{P}-\text { wave }}$ one at $11002 \mathrm{MeV}$.

\section{Conclusions}

A new era of heavy hadron spectroscopy has begun. It has been initiated experimentally by the B-factories, CLEO, BES, and the Tevatron experiments. They have provided measurements with unprecedented precision and shown evidence of new, perhaps exotic, states, and new production and decay mechanisms. It will continue with the BES upgrade, the LHC and GSI experiments and possibly with a new tau-charm factory and a super B factory. The experimental renaissance has been accompanied by an analogous one in the theory of heavy hadrons, whose language is rapidly becoming that one of effective field theories and lattice gauge theories. For many observables systematic and controlled expansions exist that lead to definite theoretical results. The construction of similar expansions for states near or above threshold still remains a challenge.

\section{Acknowledgements}

I acknowledge the financial support obtained inside the Italian MIUR program "incentivazione alla mobilità di studiosi stranieri e italiani residenti all'estero". 


\section{References}

1. N. Isgur and M. B. Wise, Phys. Lett. B 232, 113 (1989); ibid. 237, 527 (1990); Phys. Rev. Lett. 66, 1130 (1991).

2. M. Neubert, Phys. Rept. 245, 259 (1994) [arXiv:hep-ph/9306320]; A. V. Manohar and M. B. Wise, Camb. Monogr. Part. Phys. Nucl. Phys. Cosmol. 10, 1 (2000).

3. N. Brambilla et al., Heavy quarkonium physics, CERN-2005-005, (CERN, Geneva, 2005) [arXiv:hep-ph/0412158].

4. W. E. Caswell and G. P. Lepage, Phys. Lett. B 167, 437 (1986).

5. G. T. Bodwin, E. Braaten and G. P. Lepage, Phys. Rev. D 51, 1125 (1995) [Erratum-ibid. D 55, 5853 (1997)] [hep-ph/9407339].

6. A. Pineda and J. Soto, Nucl. Phys. Proc. Suppl. 64, 428 (1998) [arXiv:hepph/9707481]; N. Brambilla et al., Nucl. Phys. B 566, 275 (2000) [arXiv:hep$\mathrm{ph} / 9907240]$.

7. M. E. Luke, A. V. Manohar and I. Z. Rothstein, Phys. Rev. D 61, 074025 (2000) [arXiv:hep-ph/9910209].

8. N. Brambilla et al., Rev. Mod. Phys. 77, 1423 (2005) [arXiv:hep-ph/0410047].

9. P. Pakhlov, at ICHEP 2006.

10. W. Li, at ICHEP 2006.

11. A. Zaitsev, at ICHEP 2006.

12. R. Mussa, at ICHEP 2006.

13. N. Brambilla, Y. Sumino and A. Vairo, Phys. Lett. B 513, 381 (2001) [arXiv:hep$\mathrm{ph} / 0101305]$.

14. A. Pineda, JHEP 0106, 022 (2001) [arXiv:hep-ph/0105008].

15. M. Eidemüller, Phys. Rev. D 67, 113002 (2003) [arXiv:hep-ph/0207237].

16. A. H. Hoang and A. V. Manohar, Phys. Lett. B 633, 526 (2006) [arXiv:hep$\mathrm{ph} / 0509195]$.

17. J. H. Kühn, at ICHEP 2006.

18. N. Brambilla, Y. Sumino and A. Vairo, Phys. Rev. D 65, 034001 (2002) [arXiv:hepph/0108084].

19. A. A. Penin and M. Steinhauser, Phys. Lett. B 538, 335 (2002) [arXiv:hep-ph/0204290].

20. T. Lee, JHEP 0310, 044 (2003) [arXiv:hep$\mathrm{ph} / 0304185]$.

21. C. Contreras, G. Cvetic and P. Gaete, Phys. Rev. D 70, 034008 (2004) [arXiv:hepph/0311202].

22. B. Grinstein and Z. Ligeti, Phys. Lett. B 526, 345 (2002) [Erratum-ibid. B 601, 236
(2004)] [arXiv:hep-ph/0111392].

23. A. Pineda and A. Signer, Phys. Rev. D 73, 111501 (2006) [arXiv:hep-ph/0601185].

24. A. Petrov, at ICHEP 2006.

25. J. Brodzicka, at ICHEP 2006.

26. D. del Re, at ICHEP 2006.

27. I. Gorelov, at ICHEP 2006.

28. B. A. Petersen, at ICHEP 2006.

29. P. Kim, at ICHEP 2006.

30. R. Mizuk, at ICHEP 2006.

31. N. Brambilla and A. Vairo, Phys. Rev. D 62, 094019 (2000) [arXiv:hep-ph/0002075].

32. I. F. Allison et al. [HPQCD Collaboration], Phys. Rev. Lett. 94, 172001 (2005) [arXiv:hep-lat/0411027].

33. D. Acosta et al. [CDF Collaboration], Phys. Rev. Lett. 96, 082002 (2006) [arXiv:hepex/0505076].

34. B. A. Kniehl et al., Phys. Rev. Lett. 92, 242001 (2004) [arXiv:hep-ph/0312086]; A. A. Penin, at ICHEP 2006.

35. A. A. Penin et al., Phys. Lett. B 593, 124 (2004) [arXiv:hep-ph/0403080].

36. A. A. Penin et al., Nucl. Phys. B 699, 183 (2004) [arXiv:hep-ph/0406175].

37. A. Pineda and A. Signer, arXiv:hep$\mathrm{ph} / 0607239$.

38. N. Brambilla, Y. Jia and A. Vairo, Phys. Rev. D 73, 054005 (2006) [arXiv:hep$\mathrm{ph} / 0512369]$.

39. A. Vairo, arXiv:hep-ph/0608327.

40. W. M. Yao et al. [Particle Data Group], J. Phys. G 33, 1 (2006).

41. S. Fleming and A. K. Leibovich, Phys. Rev. D 67, 074035 (2003) [arXiv:hep$\mathrm{ph} / 0212094]$.

42. X. Garcia i Tormo and J. Soto, Phys. Rev. D 72, 054014 (2005) [arXiv:hep-ph/0507107].

43. B. Nemati et al. [CLEO Collaboration], Phys. Rev. D 55, 5273 (1997) [arXiv:hepex/9611020].

44. A. Ocherashvili et al. [SELEX Collaboration], arXiv:hep-ex/0406033; M. Mattson et al. [SELEX Collaboration], Phys. Rev. Lett. 89, 112001 (2002) [arXiv:hep-ex/0208014]; M. A. Moinester et al. [SELEX Collaboration], Czech. J. Phys. 53, B201 (2003) [arXiv:hep-ex/0212029].

45. T. Lesiak [BELLE Collaboration], arXiv:hep-ex/0605047.

46. B. Aubert et al. [BABAR Collaboration], Phys. Rev. D 74, 011103 (2006) [arXiv:hepex/0605075].

47. N. Brambilla, A. Vairo and T. Rösch, 
Phys. Rev. D 72, 034021 (2005) [arXiv:hepph/0506065].

48. S. Fleming and T. Mehen, Phys. Rev. D 73, 034502 (2006) [arXiv:hep-ph/0509313].

49. M. J. Savage and M. B. Wise, Phys. Lett. B 248, 177 (1990).

50. J. M. Flynn, F. Mescia and A. S. B. Tariq [UKQCD Collaboration], JHEP 0307, 066 (2003) [arXiv:hep-lat/0307025].

51. R. Lewis, N. Mathur and R. M. Woloshyn, Phys. Rev. D 64, 094509 (2001) [arXiv:hep$\mathrm{ph} / 0107037]$.

52. A. Ali Khan et al., Phys. Rev. D 62, 054505 (2000) [arXiv:hep-lat/9912034].

53. N. Mathur, R. Lewis and R. M. Woloshyn, Phys. Rev. D 66, 014502 (2002) [arXiv:hep$\mathrm{ph} / 0203253$.

54. J. Hu and T. Mehen, Phys. Rev. D 73, 054003 (2006) [arXiv:hep-ph/0511321].

55. Y. Jia, JHEP 0610, 073 (2006) [arXiv:hep$\mathrm{ph} / 0607290]$.

56. A. A. Penin, V. A. Smirnov and M. Steinhauser, Nucl. Phys. B 716, 303 (2005) [arXiv:hep-ph/0501042].

57. N. Brambilla and A. Vairo, Phys. Rev. D 71, 034020 (2005) [arXiv:hep-ph/0411156].

58. M. Beneke, Y. Kiyo and K. Schuller, Nucl. Phys. B 714, 67 (2005) [arXiv:hep$\mathrm{ph} / 0501289$ ].

59. M. Artuso et al. [CLEO Collaboration], Phys. Rev. Lett. 94, 032001 (2005) [hepex/0411068].

60. X. Garcia i Tormo and J. Soto, Phys. Rev. Lett. 96, 111801 (2006) [arXiv:hepph/0511167].

61. S. Gottlieb et al., PoS LAT2005, 203 (2006) [arXiv:hep-lat/0510072].

62. G. P. Lepage et al., Phys. Rev. D 46, 4052 (1992) [arXiv:hep-lat/9205007].

63. A. Gray et al., Phys. Rev. D 72, 094507 (2005) [arXiv:hep-lat/0507013].

64. N. Brambilla et al., Phys. Rev. D 63, 014023 (2001) [arXiv:hep-ph/0002250].

65. A. Pineda and A. Vairo, Phys. Rev. D 63, 054007 (2001) [Erratum-ibid. D 64, 039902 (2001)] [arXiv:hep-ph/0009145].

66. G. S. Bali, Phys. Rept. 343, 1 (2001) [arXiv:hep-ph/0001312].

67. G. Schierholz, at ICHEP 2006.

68. Y. Koma, M. Koma and H. Wittig, Phys. Rev. Lett. 97, 122003 (2006) [arXiv:heplat/0607009].

69. Y. Koma and M. Koma, arXiv:heplat/0609078.
70. W. Buchmüller, Phys. Lett. B 112, 479 (1982).

71. N. Brambilla and A. Vairo, Phys. Rev. D 55, 3974 (1997) [arXiv:hep-ph/9606344]; N. Brambilla, arXiv:hep-ph/9809263; N. Brambilla and A. Vairo, arXiv:hepph/9904330.

72. C. Patrignani [FNAL-E835 Collaboration], arXiv:hep-ex/0410085.

73. P. Rubin et al. [CLEO Collaboration], Phys. Rev. D 72, 092004 (2005) [arXiv:hepex/0508037].

74. G. T. Bodwin and A. Petrelli, Phys. Rev. D 66, 094011 (2002) [arXiv:hep-ph/0205210].

75. J. P. Ma and Q. Wang, Phys. Lett. B 537, 233 (2002) [arXiv:hep-ph/0203082].

76. N. Brambilla, E. Mereghetti and A. Vairo, JHEP 0608, 039 (2006) [arXiv:hepph/0604190].

77. N. Brambilla et al., Phys. Rev. Lett. 88, 012003 (2002) [arXiv:hep-ph/0109130].

78. N. Brambilla et al., Phys. Rev. D 67, 034018 (2003) [arXiv:hep-ph/0208019].

79. N. Brambilla et al., Phys. Lett. B 580, 60 (2004) [arXiv:hep-ph/0307159].

80. A. Vairo, Mod. Phys. Lett. A 19, 253 (2004) [arXiv:hep-ph/0311303].

81. A. Vairo, Nucl. Phys. Proc. Suppl. 115, 166 (2003) [arXiv:hep-ph/0205128].

82. E. S. Swanson, Phys. Rept. 429, 243 (2006) [arXiv:hep-ph/0601110].

83. E. Eichten et al., Phys. Rev. D 17, 3090 (1978) [Erratum-ibid. D 21, 313 (1980)].

84. E. J. Eichten, K. Lane and C. Quigg, Phys. Rev. D 69, 094019 (2004) [arXiv:hep-ph/0401210]; ibid 73, 014014 (2006) [Erratumibid. D 73, 079903 (2006)] [arXiv:hepph/0511179]; E. Eichten, at ICHEP 2006.

85. A. Le Yaouanc et al., Phys. Rev. D 8, 2223 (1973).

86. Yu. S. Kalashnikova, Phys. Rev. D 72, 034010 (2005) [arXiv:hep-ph/0506270]; Y. Kalashnikova, at ICHEP 2006.

87. K. Abe et al., arXiv:hep-ex/0507019.

88. J. L. Rosner, AIP Conf. Proc. 815, 218 (2006) [arXiv:hep-ph/0508155].

89. K. Abe et al. [BELLE Collaboration], BELLE-CONF-0331.

90. A. K. Likhoded, at ICHEP 2006

91. Y. J. Zhang and K. T. Chao, arXiv:hep$\mathrm{ph} / 0611086$.

92. S. Uehara et al. [BELLE Collaboration], Phys. Rev. Lett. 96, 082003 (2006) [arXiv:hep-ex/0512035]. 
93. K. Abe et al. [BELLE Collaboration], Phys. Rev. Lett. 94, 182002 (2005) [arXiv:hepex/0408126].

94. L. Maiani et al., Phys. Rev. D 71, 014028 (2005) [arXiv:hep-ph/0412098].

95. S. Ye, at the QWG meeting 2006.

96. X. Lou, at ICHEP 2006.

97. S. K. Choi et al. [BELLE Collaboration], Phys. Rev. Lett. 91, 262001 (2003) [arXiv:hep-ex/0309032].

98. G. Majumder, at ICHEP 2006.

99. B. Aubert et al. [BABAR Collaboration], Phys. Rev. D 71, 071103 (2005) [arXiv:hepex/0406022].

100. B. Aubert et al. [BABAR Collaboration], Phys. Rev. D 73, 011101 (2006) [arXiv:hepex/0507090].

101. D. Acosta et al. [CDF II Collaboration], Phys. Rev. Lett. 93, 072001 (2004) [arXiv:hep-ex/0312021]; G. Bauer, at the QWG meeting 2003.

102. M. Kreps, at ICHEP 2006.

103. V. M. Abazov et al. [D0 Collaboration], Phys. Rev. Lett. 93, 162002 (2004) [arXiv:hep-ex/0405004].

104. K. Abe et al. [BELLE Collaboration], arXiv:hep-ex/0408116.

105. K. Abe et al., arXiv:hep-ex/0505037.

106. G. Gokhroo et al., arXiv:hep-ex/0606055.

107. B. Aubert et al. [BABAR Collaboration], Phys. Rev. Lett. 96, 052002 (2006) [arXiv:hep-ex/0510070].

108. K. Abe et al., arXiv:hep-ex/0505038.

109. I. Kravchenko [CDF Collaboration], eConf C060409, 016 (2006) [arXiv:hep-ex/0605076].

110. M. B. Voloshin, eConf C060409, 014 (2006) [arXiv:hep-ph/0605063].

111. H. Høgaasen, J. M. Richard and P. Sorba, Phys. Rev. D 73, 054013 (2006) [arXiv:hepph/0511039]; F. Buccella et al., arXiv:hep$\mathrm{ph} / 0608001$.

112. N. A. Törnquist, Z. Phys. C 61, 525 (1994) [arXiv:hep-ph/9310247]; arXiv:hepph/0308277.

113. E. S. Swanson, Phys. Lett. B 588, 189 (2004) [arXiv:hep-ph/0311229]; ibid. 598, 197 (2004) [arXiv:hep-ph/0406080].

114. S. Pakvasa and M. Suzuki, Phys. Lett. B 579, 67 (2004) [arXiv:hep-ph/0309294].

115. M. B. Voloshin, Phys. Lett. B 579, 316 (2004) [arXiv:hep-ph/0309307]; ibid. 604, 69 (2004) [arXiv:hep-ph/0408321].

116. E. Braaten and M. Kusunoki, Phys.
Rev. D 69, 074005 (2004) [arXiv:hepph/0311147]; ibid. 72, 014012 (2005) [arXiv:hep-ph/0506087].

117. T. Skwarnicki, at ICHEP 2006.

118. B. Aubert et al. [BABAR Collaboration], Phys. Rev. Lett. 95, 142001 (2005) [arXiv:hep-ex/0506081].

119. S. Olsen, at the QWG meeting 2006.

120. Q. He [CLEO Collaboration], arXiv:hepex/0611021.

121. T. E. Coan et al. [CLEO Collaboration], Phys. Rev. Lett. 96, 162003 (2006) [arXiv:hep-ex/0602034].

122. I. Shipsey, at ICHEP 2006.

123. B. Aubert [BABAR Collaboration], arXiv:hep-ex/0607083.

124. [BELLE Collaboration], arXiv:hep-ex/0608018.

125. F. J. Llanes-Estrada, Phys. Rev. D 72, 031503 (2005) [arXiv:hep-ph/0507035].

126. C. F. Qiao, Phys. Lett. B 639, 263 (2006) [arXiv:hep-ph/0510228].

127. L. Maiani et al., Phys. Rev. D 72, 031502 (2005) [arXiv:hep-ph/0507062].

128. S. L. Zhu, Phys. Lett. B 625, 212 (2005) [arXiv:hep-ph/0507025].

129. D. Ebert, R. N. Faustov and V. O. Galkin, Phys. Lett. B 634, 214 (2006) [arXiv:hepph/0512230].

130. X. Liu, X. Q. Zeng and X. Q. Li, Phys. Rev. D 72, 054023 (2005) [arXiv:hepph/0507177].

131. C. Z. Yuan, P. Wang and X. H. Mo, Phys. Lett. B 634, 399 (2006) [arXiv:hepph/0511107].

132. E. Kou and O. Pene, Phys. Lett. B 631, 164 (2005) [arXiv:hep-ph/0507119].

133. F. E. Close and P. R. Page, Phys. Lett. B 628, 215 (2005) [arXiv:hep-ph/0507199].

134. K. J. Juge, J. Kuti and C. Morningstar, Phys. Rev. Lett. 90, 161601 (2003) [arXiv:hep-lat/0207004].

135. W. S. Hou, Phys. Rev. D 74, 017504 (2006) [arXiv:hep-ph/0606016] and at ICHEP 2006.

136. M. T. AlFiky, F. Gabbiani and A. A. Petrov, Phys. Lett. B 640, 238 (2006) [arXiv:hep-ph/0506141].

137. T. Manke et al. [CP-PACS Collaboration], Phys. Rev. D 64, 097505 (2001) [arXiv:heplat/0103015]. 